\title{
Extremely long transition phase of thermal convection in the mantle of massive super-Earths
}

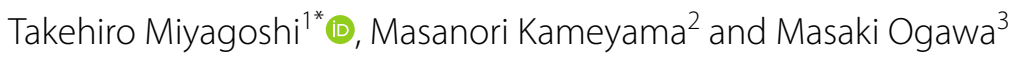

\begin{abstract}
Adiabatic compression is a key factor that exerts control over thermal convection in the compressible solid mantle of super-Earths. To discuss the effects of adiabatic compression, we present a numerical model of transient convection in the cooling mantle of a super-Earth that is ten times larger in size than the Earth. The calculations started with the shallow mantle that was hotter than expected by the extrapolation from the deep mantle conditions. This type of initial thermal state of the mantle is expected to naturally occur in real super-Earths due to heating by giant impacts at the time of their formation. With our initial setup conditions, the convection temporarily occurs as a layered convection for the first several to ten billion years of the calculation and then changes its style into a whole layer convection. The long duration of the transient stage suggests that mantle convection currently occurs as a temporal layered convection in many of the super-Earths. A temporal layered convection, if it occurs, can exert control over the tectonic activities of super-Earths. Future studies should clarify how internal heating and complicated rheological properties of mantle materials including their pressure dependence affect the duration of the temporal layered convection.
\end{abstract}

Keywords: Super-Earths, Thermal convection, Mantle, Thermal evolution

\section{Introduction}

Dynamics of the mantle in super-Earths is a key issue in studying their thermal history and surface environments. Stimulated by the detection of a large number of super-Earths, which are extrasolar terrestrial planets with a mass of up to ten times the Earth's (e.g., Borucki et al. 2011), many theoretical and numerical studies have been conducted to explain the structure and dynamics of their interior. In many of these studies, mantle convection is modeled by the parameterized convection method (e.g., Valencia and O'connell 2009; Stamenković et al. 2011) or by a steady convection of a Boussinesq fluid where the effect of adiabatic compression is neglected (e.g., van Heck and Tackley 2011; Foley et al. 2012; Stein et al. 2013). Our studies (Miyagoshi et al. 2014, 2015; Tachinami et al. 2014; Kameyama et al. 2015), however, report

\footnotetext{
*Correspondence: miyagoshi@jamstec.go.jp

${ }^{1}$ Department of Deep Earth Structure and Dynamics Research, Japan Agency for Marine-Earth Science and Technology, 3173-25 Showa-machi, Kanazawa-ku, Yokohama, Kanagawa 236-0001, Japan

Full list of author information is available at the end of the article
}

that adiabatic compression significantly affects thermal convection in massive super-Earths by greatly reducing the efficiency of convective heat transport and also by reducing the activity of hot plumes ascending from the bottom boundary of the mantle. A simple extrapolation of mantle convection in the Earth and other planets of our solar system to super-Earths is unreliable.

In the course of these numerical studies of mantle convection in super-Earths, we noticed that the nature of the thermal convection substantially changes with time, as the convection approaches the statistical steady state, and that the period of the transitional stage becomes as long as ten billion years based on the parameter values of massive super-Earths; the length of the transitional stage is comparable to the age of the universe. This finding suggests that the evolution of super-Earths may be strongly dependent on their initial conditions determined by the process of planetary formation. Therefore, caution may be necessary when numerically simulating mantle evolution in super-Earths. In their numerical models of statistically steady thermal convection of a temperature- and 
pressure-dependent viscosity fluid that yields at high stress, Tackley et al. (2013) found that plume-like hot regions arise from the mid-mantle and that subducting slabs stop sinking there. Noack and Breuer (2014) conclude from their calculation of similar models of convection that evolves with time over a 2.5-Gyr time span that the pressure dependence of viscosity critically affects the operation of plate tectonics in super-Earths. Given the long time span needed for the effects of the initial conditions to disappear, caution is necessary to apply these numerical results to super-Earths.

The initial temperature distribution in super-Earths is determined by the formation process of the planets. In the first runaway growth stage of the formation process of a planet (e.g., Wetherill and Stewart 1989), planetesimals collide into the surface of the protoplanet and the surface and the shallow mantle are strongly heated by released gravitational energy. At the end of the later oligarchic growth stage (e.g., Chambers 2001; Kokubo and Ida 1998), giant impacts induce strong shock waves within the planets to raise the temperature most prominently at the surface and the core-mantle boundaries where there is a strong density increase that causes shock wave reflection (e.g., Canup 2004). In both stages, the temperature in the shallower mantle is likely to become higher than that in the deep mantle, and the excess temperature of the shallow mantle can be as high as several thousands of degrees [Fig. 4 of Canup (2004)]. The excess temperature may survive the temperature change in the mantle subsequently caused by core formation and heat transport in the magma ocean.

In this study, we address the issue of the potential excess temperature in the shallow mantle exerting control over the subsequent thermal evolution of massive super-Earths. For this purpose, we present an example of transient mantle convection we encountered in our numerical studies of a massive super-Earth to discuss how the nature of thermal convection can change in the course of its approach to the statistical steady state and on what timescale the change occurs (note that Miyagoshi et al. (2015) reports only the convections in their statistical steady states).

\section{Model description}

Since the model and basic equations adopted here are the same as those implemented in our previous studies (Miyagoshi et al. 2014, 2015), we only briefly summarize them here. The compressible Navier-Stokes equation with the infinite Prandtl number, the mass conservation equation, and the heat transport equation are solved in a two-dimensional rectangular box with an aspect ratio of four under the truncated anelastic liquid approximation using the numerical simulation code ACuTEMan
(Kameyama 2005; Kameyama et al. 2005). The absolute temperature is fixed at $T_{\mathrm{s}}$ and $T_{\mathrm{b}}$ at the top and bottom boundaries, respectively, while there is no heat flux on the sidewalls. We neglected the possible effects of decreasing $T_{\mathrm{b}}$ with time due to core cooling on the dynamics of the compressible mantle, as further discussed in "Discussion" section. All boundaries are shear stress-free and impermeable.

The viscosity strongly depends on temperature:

$$
\eta=\eta_{b} \exp \left[E\left(T_{b}-T\right)\right]
$$

where $\eta_{\mathrm{b}}$ is the viscosity at the bottom boundary and $E$ is a constant, called the Frank-Kamenetskii approximation parameter. We consider the temperature dependence of viscosity in the calculations because it is the agent that induces the lithosphere, a prominent feature of mantle convection. This approximation gives a fairly good estimate of the temperature-dependent viscosity beneath the stagnant lithosphere, as discussed in Appendix A of Reese et al. (1999). We ignore, however, the pressure dependence of viscosity, considered in earlier models (e.g., Tackley et al. 2013), to make the effects of adiabatic compression conspicuous (see "Discussion" section for further discussion). We set the ratio in viscosity between the top and bottom boundaries

$$
\Delta \eta=\exp \left[E\left(T_{\mathrm{b}}-T_{\mathrm{s}}\right)\right]
$$

at $10^{6}$

The basic equations in their non-dimensional forms are

$$
\begin{aligned}
& \nabla^{*} \cdot\left[\bar{\rho}^{*}\left(z^{*}\right) \mathbf{u}^{*}\right]=0, \\
& -\nabla^{*} p^{*}+\nabla^{*} \cdot \tau^{*}+R a^{*} \bar{\rho}^{*}\left(z^{*}\right) \alpha^{*}\left(z^{*}\right) T^{*} \mathbf{e}_{\mathbf{z}}=0, \\
& \tau_{i j}^{*}=\eta^{*}\left(T^{*}\right)\left[\left(\nabla^{*} \mathbf{u}^{*}+{ }^{\mathbf{t}} \nabla^{*} \mathbf{u}^{*}\right)_{i j}-\frac{2}{3} \nabla^{*} \cdot \mathbf{u}^{*} \delta_{i j}\right], \\
& \bar{\rho}^{*}\left(z^{*}\right)\left[\frac{\mathrm{d} T^{*}}{\mathrm{~d} t^{*}}+D i^{*} \cdot \alpha^{*}\left(z^{*}\right) w^{*} T^{*}\right] \\
& \quad=\nabla^{* 2} T^{*}+\frac{D i^{*}}{R a^{*}} \tau_{i j}^{*} \frac{\partial u_{i}^{*}}{\partial x_{j}^{*}} .
\end{aligned}
$$

The quantities with asterisks are non-dimensional. Here, $\mathbf{u}$ and $\mathbf{e}_{\mathbf{z}}$ are the fluid velocity and the vertical unit vector, $p$ is non-hydrostatic pressure, $w$ is the vertical component of fluid velocity, and $\delta_{i j}$ is Kronecker's delta. These equations are non-dimensionalized by the length scale of $d$ (the depth of the mantle), the timescale of $d^{2} / \kappa$ (where $\kappa=10^{-6} \mathrm{~m}^{2} / \mathrm{s}$ is the thermal diffusivity), and the temperature scale of $\Delta T \equiv T_{\mathrm{b}}-T_{\mathrm{s}}$. Here, $T^{*}=T / \Delta T$ is the normalized absolute temperature rather than the relative temperature $\left(T-T_{\mathrm{s}}\right) / \Delta T$ that is often adopted in Boussinesq models. The viscosity $\eta$, the thermal 
expansivity $\alpha$, and the density $\rho$ are normalized by their values at the surface boundary $\eta_{\mathrm{s}}=\eta_{\mathrm{b}} \Delta \eta, \alpha_{0}$, and $\rho_{0}$, respectively. The time derivative in Eq. (6) is Lagrangian. Hereinafter, the values are non-dimensional unless otherwise stated and we drop the asterisks. The units of normalization are summarized in Table 1. The mantle depth $d$ is calculated from the scaling law suggested by Valencia et al. (2007) as $d=d_{\oplus}\left(M_{p}\right)^{0.28}$ where $d_{\oplus}$ is the manthe depth of the Earth $(\sim 2900 \mathrm{~km})$ and $M_{\mathrm{p}}(=10$ in this paper) is the planetary mass normalized by the Earth's mass.

In the basic equations, we assume a simple linear dependence of the reference density $\bar{\rho}$ on the depth $1-z$ ( $z$ is the height from the bottom),

$$
\bar{\rho}(z)=1+\left[1.75\left(M_{\mathrm{p}}\right)^{0.258}-1\right](1-z),
$$

based on the earlier models of mantle structure (Valencia et al. 2006). On the other hand, the thermal expansivity $\alpha$ depends on the depth as shown in Fig. 1a. The dependence is the same as the one in our previous studies (Tachinami et al. 2014; Miyagoshi et al. 2014; 2015); $\alpha$ decreases by a factor of 10 from the surface to the bottom boundary. This profile is estimated for $\mathrm{MgO}$ in a former ab initio calculation (Tsuchiya and Tsuchiya 2011) and is consistent with the previous laboratory measurements under high pressure (Chopelas and Boehler 1992).

There are three free parameters in the formulation: the Rayleigh number $R a=\rho_{0} \alpha_{0} g \Delta T d^{3} / \eta_{s} k$ where $g$ is the gravitational acceleration; $T_{\mathrm{s}} / \Delta T$ is the normalized surface temperature; $D i=\alpha_{0} g d / C_{\mathrm{p}}$ is the dissipation number where $C_{\mathrm{p}}$ is the specific heat. The numerical results depend on the surface temperature $T_{\mathrm{s}} / \Delta T$ in our models in contrast to Boussinesq models since the magnitude of the effects of adiabatic compression is proportional to the absolute temperature [see the second term of the lefthand side of Eq. (6)]. The normalized bottom temperature is calculated from $T_{\mathrm{s}} / \Delta T$ as $T_{\mathrm{b}} / \Delta T=T_{\mathrm{s}} / \Delta T+1$ and hence is not an independent free parameter.

We adopt $R a=10^{4}, T_{\mathrm{s}} / \Delta T=0.1$, and $D i=5$, which correspond to a planet of ten times the Earth's mass.

Table 1 The scaling unit for non-dimensionalization

\begin{tabular}{lll}
\hline The scaling unit & Symbol & Value \\
\hline Length (mantle depth) & $d$ & $5500(\mathrm{~km})$ \\
Thermal diffusivity & $k$ & $10^{-6}\left(\mathrm{~m}^{2} / \mathrm{s}\right)$ \\
Time & $d^{2} / \mathrm{k}$ & $3.0 \times 10^{19}(\mathrm{~s}) \sim 950 \mathrm{Gyr}$ \\
Velocity & $\mathrm{K} / d$ & $5.7 \times 10^{-4}(\mathrm{~cm} /$ year $)$ \\
Thermal expansivity & $a_{0}$ & $4 \times 10^{-5}\left(\mathrm{~K}^{-1}\right)$ \\
Temperature & $\Delta T$ & $3000(\mathrm{~K})$ \\
\hline
\end{tabular}
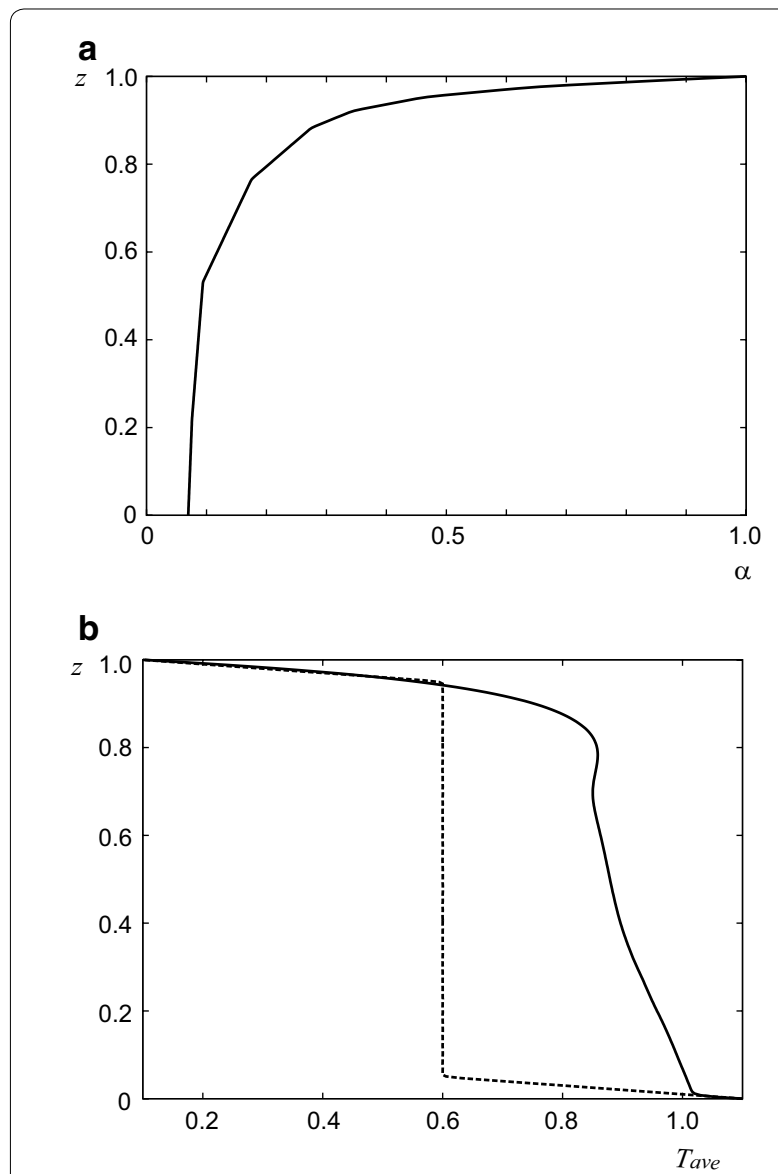

Fig. 1 a The plot of the normalized thermal expansion coefficient $a$ (horizontal axis) against height $z$ (vertical axis). b The solid line shows the horizontally averaged initial temperature profile plotted against height $z$. As for the dashed line, see the text

These values are the same as the values for Case 5 in Table 1 of Miyagoshi et al. (2015). Note that the free parameters are all defined with the surface values of material properties. The Rayleigh number becomes $10^{10}$ when it is defined with the viscosity at the bottom boundary, as calculated from the assumed value of $\Delta \eta$. The magnitude of the effects of adiabatic compression is proportional to $\alpha^{*}\left(z^{*}\right) D i$ rather than $D i$ at depth $z$, as shown in Eq. (6), and the effective dissipation number, i.e., the dissipation number averaged over the entire mantle becomes 0.79:

$$
\overline{D i}=D i \int_{0}^{1} \alpha \mathrm{d} z
$$

This value is close to the one assumed in an earlier model (Tackley et al. 2013).

We started the calculation from the initial temperature profile illustrated by the solid line in Fig. 1b. We obtained this initial temperature profile as follows: We 
first assumed that the non-dimensional temperature is constant at $\left(T_{\mathrm{s}}+T_{\mathrm{b}}\right) / 2=0.6$ over the entire mantle except along the boundaries (see the dotted line in Fig. 1b) and started to calculate convection. Then, we uniformly heated the mantle, until the average temperature of the whole mantle became 1.05 times that at the equilibrium state. Starting from this temperature profile, we observed the evolution of thermal convection in the cooling mantle.

\section{Results}

In this section, we present the overall structure of thermal convection and its development with time in Figs. 2 and 3 . Figure 2 shows the distribution of temperature and convective flow (left column) and the plots of horizontally averaged temperature against height (right column) at each time interval. Figure 3 shows the distribution of potential temperature $T_{\mathrm{p}}(x, z)$ calculated from Fig. 2 as

$$
T_{\mathrm{p}}(x, z)=T(x, z) \exp \left(-D_{i} \int_{z}^{1} \alpha(\xi) \mathrm{d} \xi\right) .
$$

We present the potential temperature as in Tachinami et al. (2014) and Miyagoshi et al. (2015) because it is more relevant to mantle convection than the temperature itself. Temperature variation caused by adiabatic compression does not drive the mantle convection; nevertheless, it overshadows the potential temperature variation that drives the mantle convection at large $D i$ values assumed here (Fig. 2).

At the initial transient stage (panels a-d in Figs. 2, 3), the convection is layered. In the upper layer, convection starts at $t \sim 0.156 \times 10^{-2}$ when the thermal boundary layer (TBL) along the surface boundary becomes sufficiently thick owing to the conductive cooling from the boundary; cold plumes begin to descend from the TBL. Plume heads take a time on the order of $0.9 \times 10^{-2}$ to sink to the layering boundary (panels a-d in Figs. 2, 3). In contrast, hot ascending plumes have been prominent in the lower layer since the beginning of the calculation, as shown in Fig. 3 (the panels a-d); low viscosity that results from high temperature (Fig. 2) is the reason for the higher plume activity. Indeed, the fluid velocity is higher in the lower layer (see the arrows in Fig. 2). The heads of the hot plumes ascend to the layering boundary at the height of $z \sim 0.5$ where the temperature of the plume head decreases to the ambient temperature owing to adiabatic decompression. The boundary hardly moves throughout the initial stage.

Figures 3 and 4 show why convection is layered in this stage. Although the temperature in the upper layer is lower than that in the lower layer (Fig. 2), the potential temperature in the upper layer is higher than that in the lower layer. The difference in the potential temperature between the two layers decreases with time owing to the cooling from the surface boundary and heating from the bottom boundary, but the upper layer is still slightly hotter until $t \sim 1.2 \times 10^{-2}$.

The initial stage of convection ends at $t \sim 1.2 \times 10^{-2}$ (about 11.4 billion years in dimensional quantity). After that, cold plumes penetrate through the layering boundary and the convective structure changes to a whole layer one (panel $\mathrm{f}$ in Figs. 2, 3). The distribution of horizontally averaged temperature becomes close to the adiabatic one in the entire convecting mantle (from panels $g$ to 1 in Fig. 2). Whole layer convection is the convective flow pattern that we obtained as the statistical steady state in Miyagoshi et al. (2015).

Effects of the transition from layered convection to whole layer convection are observed in the plot of the root-mean-square (RMS) fluid velocity, $V_{\mathrm{RMS}}$, as shown in Fig. 5b. The $V_{\text {RMS }}$ rapidly increases at $t$ around $1.2 \times 10^{-2}$ and remains high after that. In contrast, the transition does not noticeably affect the heat flux at the surface (Fig. 5a). The surface heat flux gradually decreases with time because of the thickening of the surface TBL. The heat flux at the surface is larger than that at the bottom boundary after $t=0.004$, and the mantle cools during that period of time as a whole. After the transition, the gradual cooling from the surface continues, until the heat flux at the bottom increases and that at the surface decreases to the common equilibrium value of 3.5.

Figure 6 presents the nature of the transition from layered convection to whole layer convection in more detail. In this figure, we show $-\left(\mathrm{d} T_{\text {ave }} / \mathrm{d} z\right)$ (red curves), the adiabatic temperature gradient calculated from $-\left(\mathrm{d} T_{\mathrm{ave}} \mathrm{d} z\right)_{\mathrm{ad}}=\alpha \cdot D i \cdot T_{\mathrm{ave}}$ (blue curves), and the plots of the horizontally averaged RMS velocity $V_{\text {RMS } \_}(z)=\sqrt{\left\langle V_{x}^{2}(x, z)+V_{z}^{2}(x, z)\right\rangle_{\text {ave } \_x}}$, where $T_{\text {ave }}$ is the horizontally averaged temperature, $V_{x}$ and $V_{z}$ are the $x$ - and $z$-components of the velocity, and \langle\rangle$_{\text {ave } \_x}$ means the average in $x$ (horizontal) direction. All of the quantities are plotted against height during the period when the convection is layered. The left column shows that the two temperature gradients, $-\left(\mathrm{d} T_{\text {ave }} / \mathrm{d} z\right)$ and $-\left(\mathrm{d} T_{\text {ave }} /\right.$ $\mathrm{d} z)_{\mathrm{ad}}$, coincide with each other in the lower layer where plumes are prominent throughout the initial transient stage. In the upper layer, however, the two gradients substantially deviate from each other. The black dashed lines in the left column show the depth where the difference $\left[-\left(\mathrm{d} T_{\text {ave }} / \mathrm{d} z\right)_{\text {ad }}\right]-\left[-\left(\mathrm{d} T_{\text {ave }} / \mathrm{d} z\right)\right]$ takes its maximum value. The descent of cold plume heads is most strongly impeded at this depth, and $V_{\text {RMS }}$ takes its local minimum value. Indeed, the dashed lines indicate the maximum depth of cold descending plume heads. The transition from layered convection to whole layer convection occurs when the 

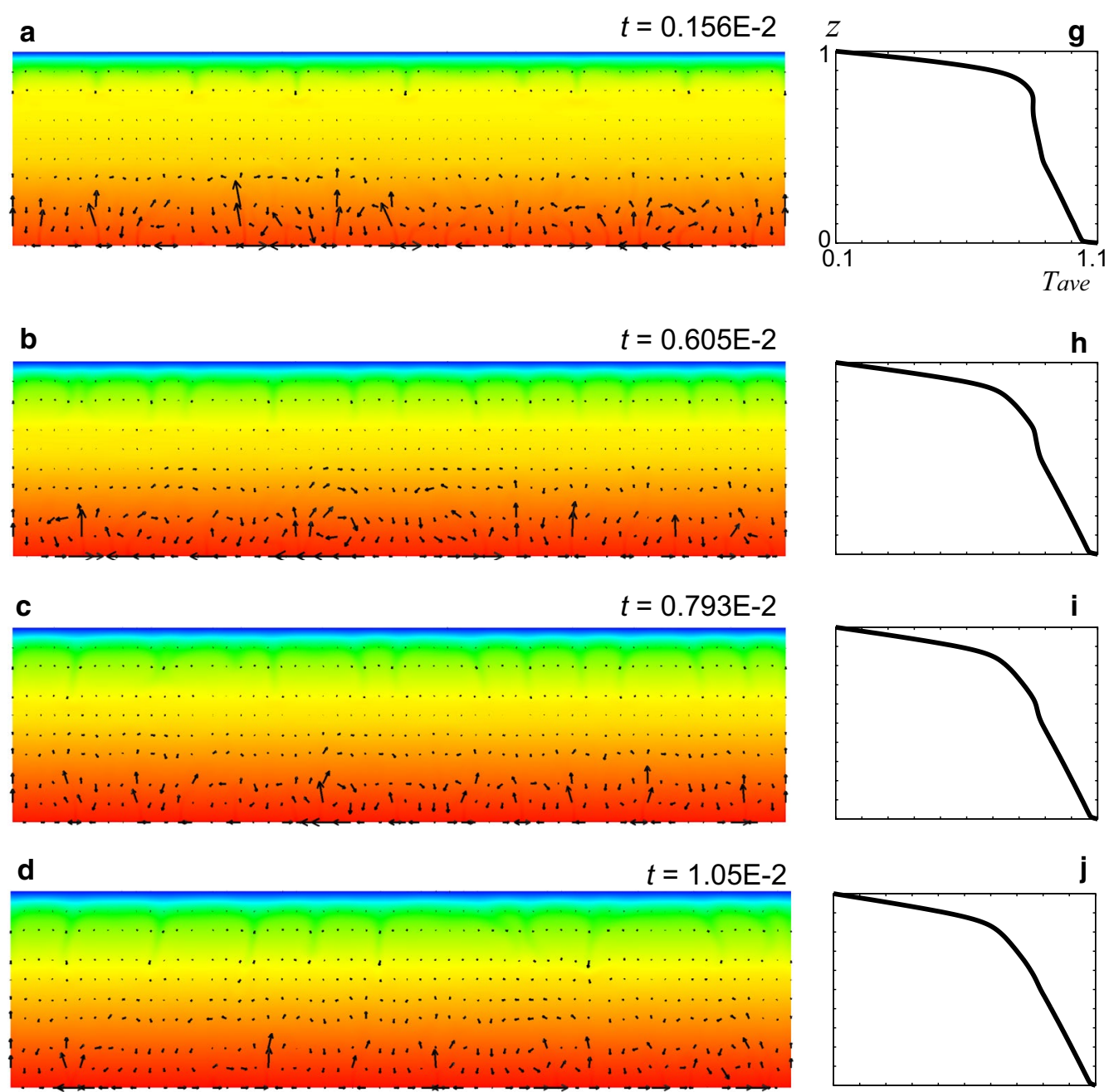

e

$t=1.20 \mathrm{E}-2$
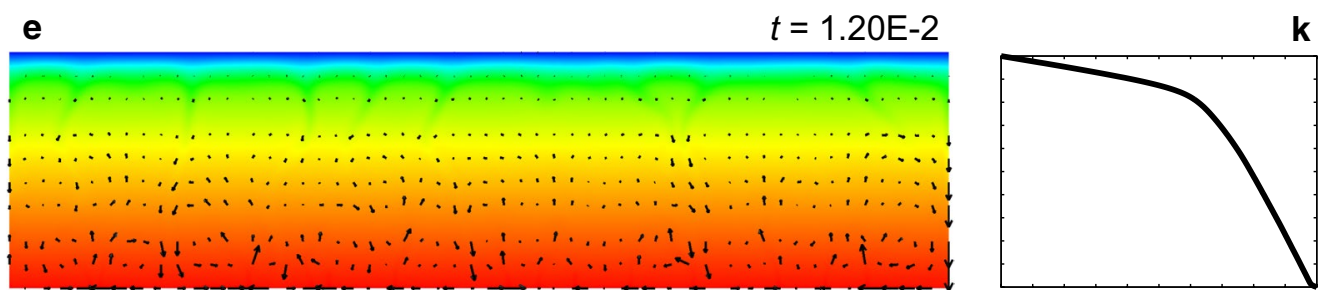

$\mathbf{f}$

$t=1.34 \mathrm{E}-2$

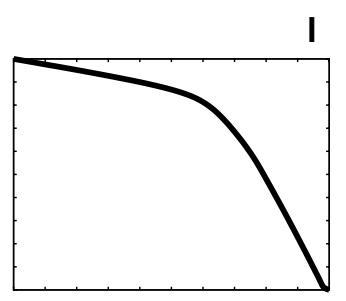

$\longrightarrow=4000$

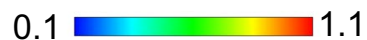

Fig. 2 Snapshots of the calculated temperature distribution (left column) and its horizontal average $T_{\text {ave }}$ plotted against height $z$ (right column) taken at time $t=0.156 \mathrm{E}-2$ (panels $\mathbf{a}$ and $\mathbf{g}$ ), 0.605E-2 (panels $\mathbf{b}$ and $\mathbf{h}$ ), $0.793 \mathrm{E}-2$ (panels $\mathbf{c}$ and $\mathbf{i}$ ), 1.05E-2 (panels $\mathbf{d}$ and $\mathbf{j}$ ), 1.20E-2 (panels e and $\mathbf{k}$ ), 1.34E-2 (panels $\mathbf{f}$ and $\mathbf{I}$ ) 


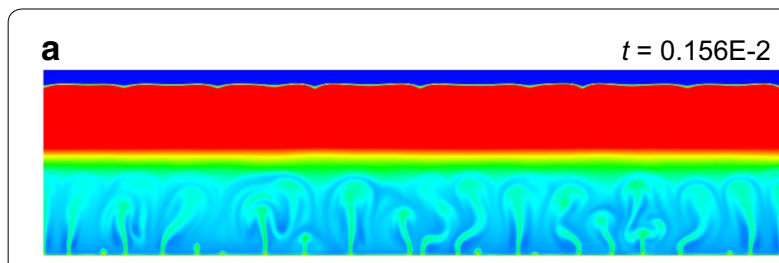

b
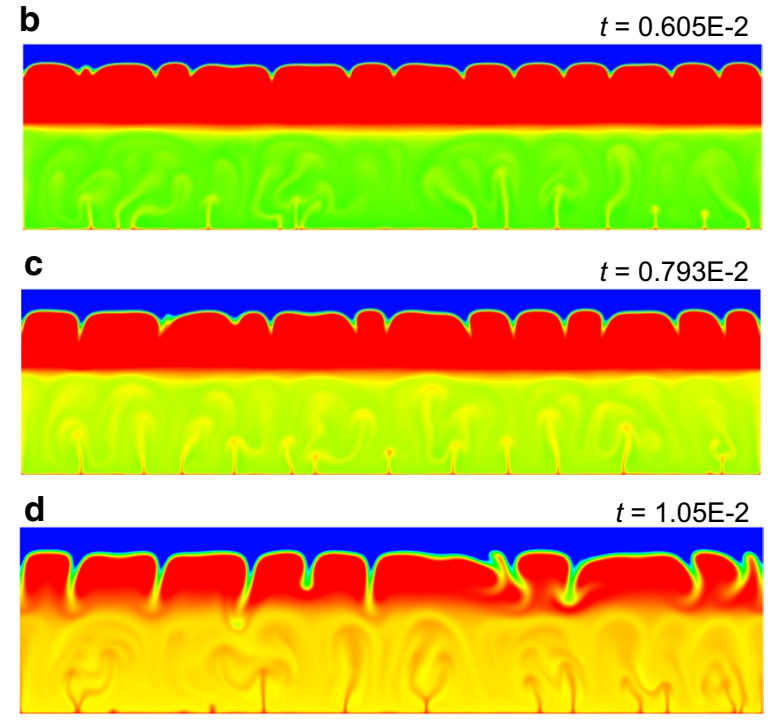

e

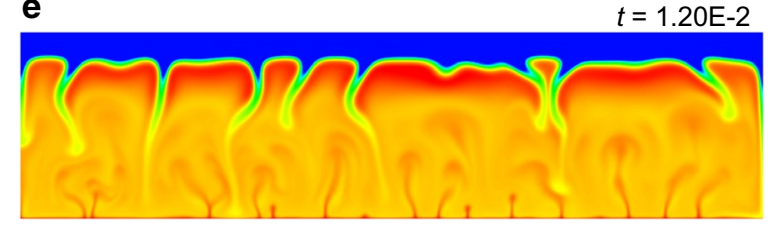

f

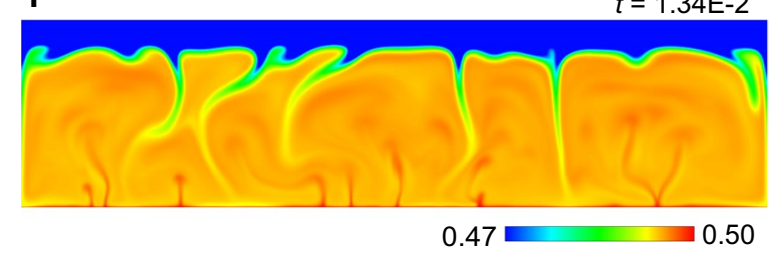

Fig. 3 Snapshots of the potential temperature distributions that correspond to the temperature distributions shown in Fig. 2, taken at time $t=0.156 \mathrm{E}-2$ (panel a), 0.605E-2 (panel b), $0.793 \mathrm{E}-2$ (panel $\mathbf{c}$ ), 1.05E-2 (panel d), 1.20E-2 (panel e), 1.34E-2 (panel f)

depth indicated by the dashed line reaches the boundary at the height of $z \sim 0.5$ (Fig. 3).

\section{Discussion}

Our numerical models show that in the mantle of many of massive super-Earths, a layered convection can temporarily occur and continue for several billion years if the shallow mantle is hotter than expected by the adiabatic extrapolation from deep mantle as a consequence of the formation process of the planets. The thermal and dynamic state of the interior may be in the transient stage, even today, in super-Earths. The transient-layered convection, if it occurs, would substantially affect the style of tectonic activities on the planet. The occurrence of plate tectonics on super-Earths may not be solely determined by the planet's size and surface environment (e.g., Valencia et al. 2007; Valencia and O'Connell 2009; Korenaga 2010; van Heck and Tackley 2011; Foley et al. 2012; Stein et al. 2013; Stamenković and Breuer 2014). It may also depend on whether or not the convection is in its initial transient stage.

Because of its potential effects on tectonic evolution, it is important to understand how long initial layered convection can continue in a super-Earth. In our numerical model, the transition to the whole layer convection occurs when the cold plume's head descends to the layering boundary (Fig. 3). The cold plume heads further penetrate into the deep mantle because their potential temperature is lower than the average potential temperature of the deep mantle and hence the cold plume heads are denser than the average lower mantle materials when they are brought into the lower mantle. Note that the average potential temperature in the shallower mantle is still slightly higher than that of the deep mantle and that the mantle is still stably stratified as a whole at the moment of the transition. Although the convective instability at the base of the lithosphere takes some time, on the order of $10^{-3}$ (Fig. 3a) to induce cold plumes, most of the duration of the layered convection (0.012) is spent for cold plumes to move downward to the layering boundary. Figure 6 suggests that the duration is scaled with the overturn time of mantle convection in the shallow mantle: In the shallow mantle, the RMS average velocity $V_{\text {RMS }}$ is about 100 (see Fig. 6). The overturn time of convection $\tau$ defined by $d_{\mathrm{s}} / V_{\mathrm{RMS}}$ where $d_{\mathrm{s}}$ is the depth of the shallow mantle beneath the lithosphere (about 0.3, Fig. 6) is, therefore, 0.003 , and the duration of the layered convection is $4 \tau$.

The duration would be shorter at a higher Rayleigh number because of the shorter turnover time of convection. On the contrary, a higher surface temperature suggested for some super-Earths (Léger et al. 2009) may extend the duration, because a higher surface temperature reduces the vigor of the convection driven by cooling from the surface boundary and hence makes the turnover time of convection longer (Tachinami et al. 2014).

There are many other factors that can affect the duration but are neglected for simplicity in this study. One of such factors is internal heating. The duration would be shorter if the deep mantle contains an internal heat source that reduces the excess temperature of the shallow mantle (i.e., the potential temperature in the shallow mantle minus that in the deep mantle). One possible internal heat source is radioactive elements. If the 


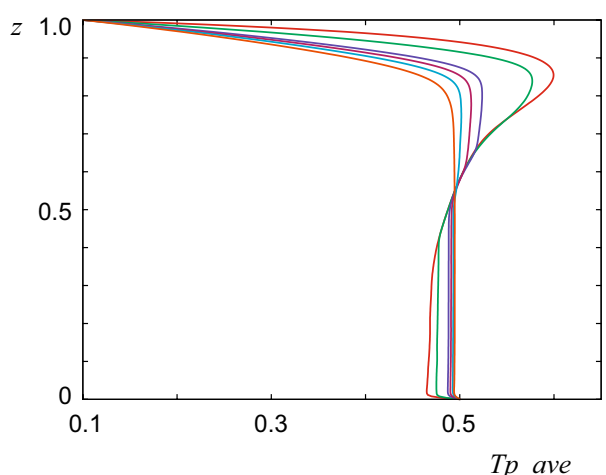

Fig. 4 Development of horizontally averaged potential temperature $T_{p-a v e}$ against height $z$. The red, green, blue, purple, light blue, and orange lines show the plots taken at $t=0,0.156 \times 10^{-2}, 0.605 \times 10^{-2}$, $0.793 \times 10^{-2}, 1.01 \times 10^{-2}$, and $1.34 \times 10^{-2}$, respectively

amount of content of radioactive elements is equal to that of the Earth, these elements would raise the temperature by about $2000 \mathrm{~K}$ over 4.5 Gyr (e.g., Turcotte and Schubert 2014). This temperature increase is a substantial fraction of the excess temperature of the shallow mantle expected to be induced by a giant impact on the Earth (e.g., Canup, 2004) and may not be negligibly small even for a superEarth. Tidal heating due to the central star can be, however, more important. In an extrasolar terrestrial planet GJ876d (Jackson et al. 2008) for which the semi-major axis of the orbit is $0.0208 \mathrm{AU}$, for example, the tidal heating is suggested to be equivalent of $10^{4}-10^{5} \mathrm{~W} \mathrm{~m}^{-2}$ surface heat flux, about $10^{5}$ times the surface heat flux of the Earth. In such a planet, the duration of the transient-layered mantle convection would be negligibly short in its evolutionary history.

The potential influence of the bottom thermal boundary condition on convection is also worth discussing. We assumed that the temperature is fixed at the bottom boundary; however, the temperature may decrease with time in super-Earths because of core cooling. Given the low efficiency of convective cooling of the compressible mantle of super-Earths found in Miyagoshi et al. (2015), the amount of the temperature decrease is probably small and is unlikely to substantially affect the duration of the transient-layered convection.

It is difficult to predict the effects of the pressure- or depth-dependent viscosity, which are neglected in this study, on the duration. A previous one-dimensional parameterized convection model (Stamenković et al. 2012) suggests that pressure-dependent viscosity makes the duration of the transient stage longer. The mixing length theory (Tachinami et al. 2011) and two-dimensional statistically steady models (Tackley et al. 2013) suggest that high viscosity in the deep mantle due to its pressure dependence or the post-perovskite transition reduces the activity of convection in contrast to our model suggesting that a vigorous convection occurs from the beginning of the calculated time span. The suggested lower activity of the deep convection layer would affect the duration of the transient-layered convection. Whether the duration becomes longer or shorter, however, depends on the internal heating rate and other factors.

Can a lateral thermal heterogeneity overlaid on the initial temperature distribution affect the subsequent transient-layered convection? When a giant impact occurs, a large lateral contrast arises in the temperature distribution of the mantle (e.g., Canup 2004). In the past numerical studies of Mars (Roberts et al. 2009) and Mercury (Roberts and Barnouin 2012), an initial temperature anomaly caused by a giant impact laterally spread in only 10-100 Myr. This characteristic time is shorter than the turnover time of mantle convection expected for superEarths, and the influence of initial lateral heterogeneities is probably small.
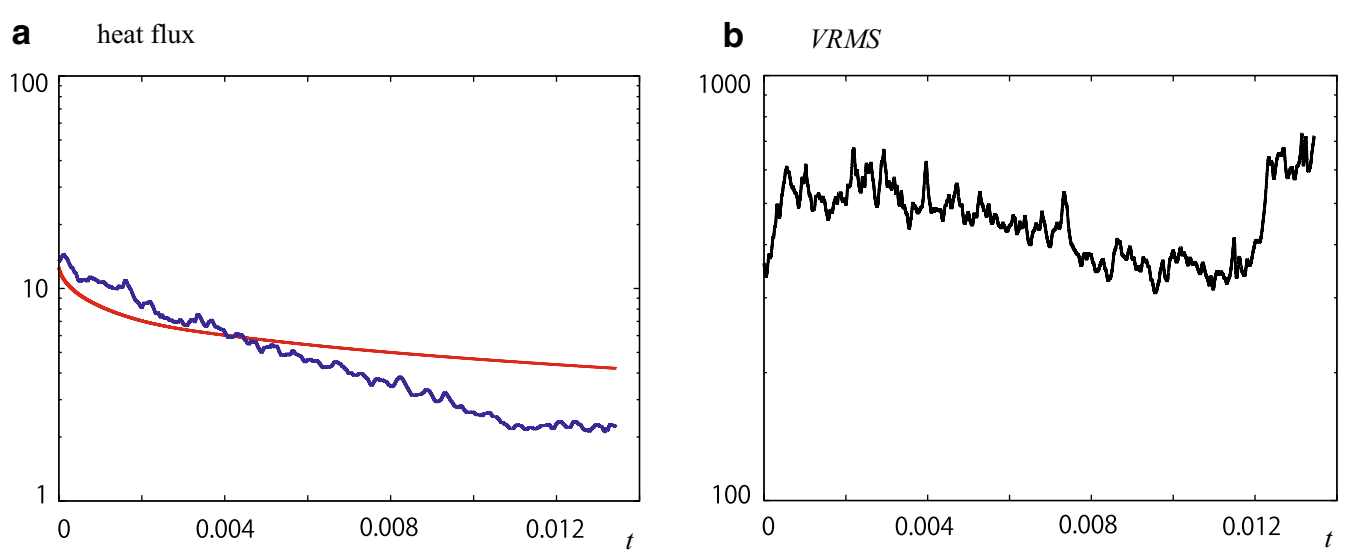

Fig. 5 a The average heat flux at the surface of the planet (red) and the core-mantle boundary (blue), both plotted against time. $\mathbf{b}$ The RMS-averaged velocity plotted against time 


\section{$t=0.156 \mathrm{E}-2$}
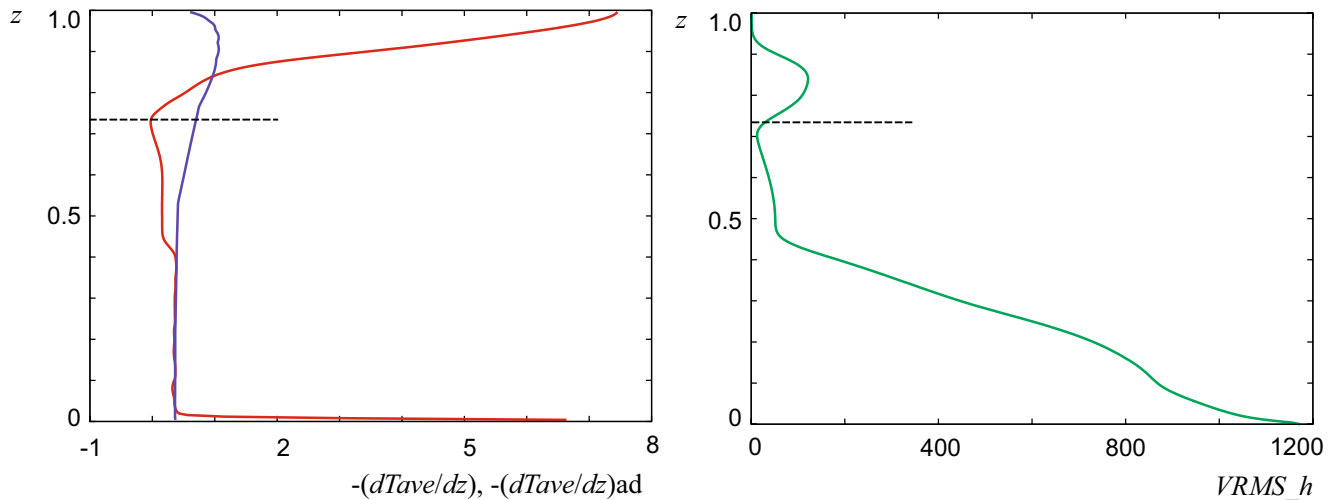

$t=0.414 \mathrm{E}-2$
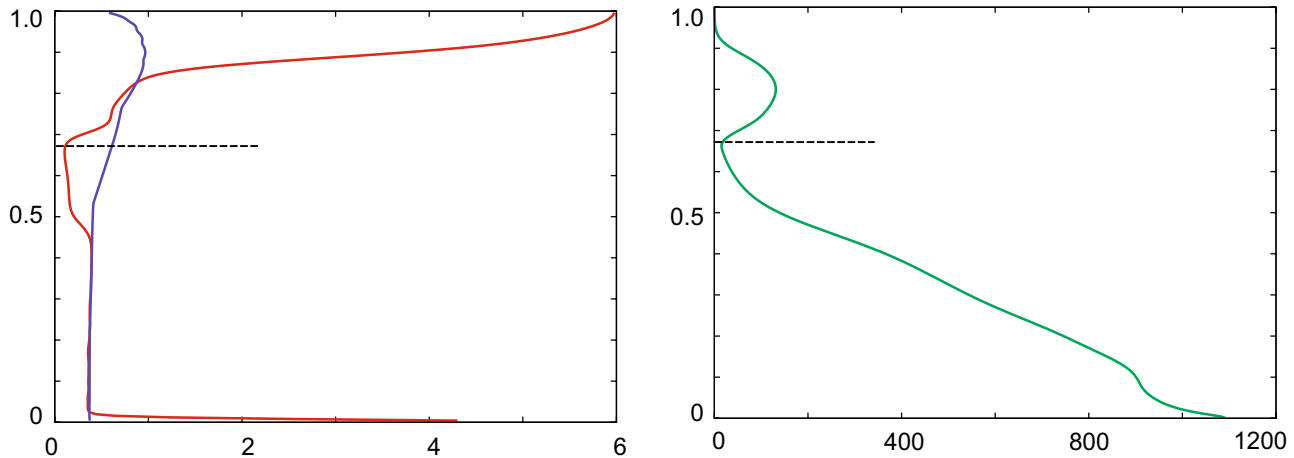

$t=0.605 \mathrm{E}-2$
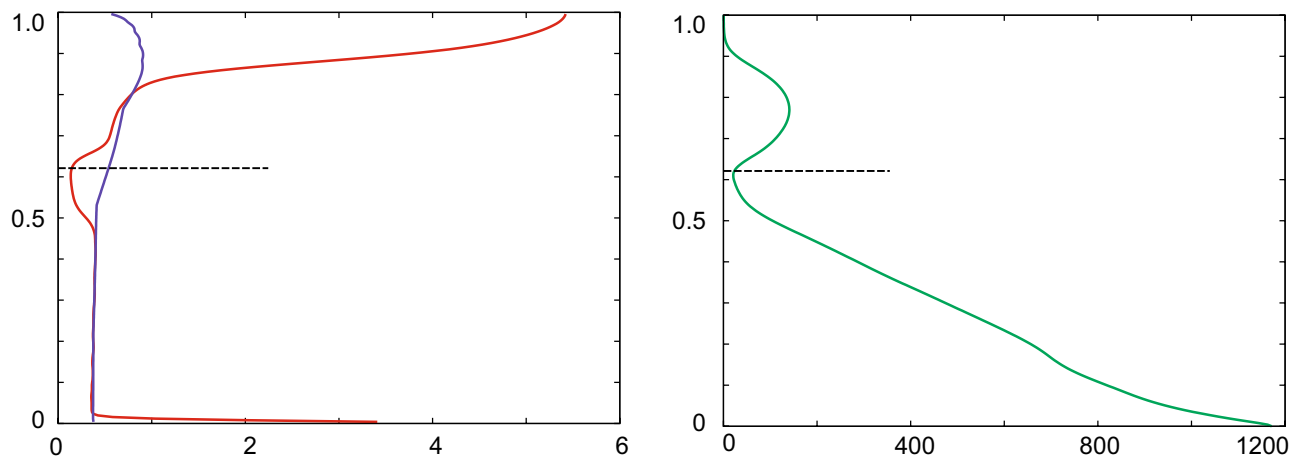

$t=0.793 \mathrm{E}-2$
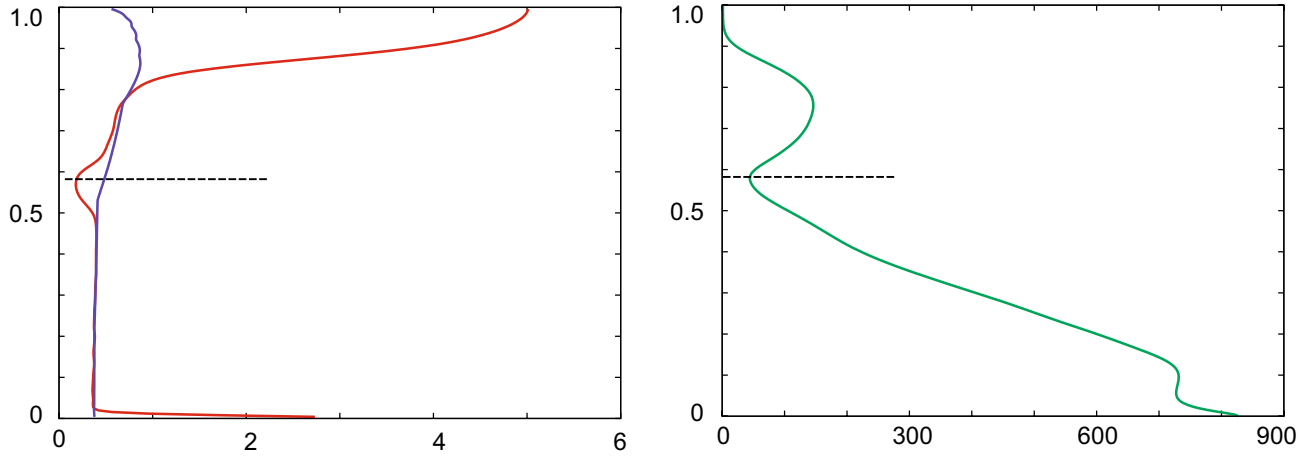

Fig. 6 The gradient of horizontally averaged temperature $-\left(\mathrm{d} T_{\mathrm{ave}} / \mathrm{d} z\right)$ (red curves), the adiabatic temperature gradient calculated from $-\left(\mathrm{d} T_{\text {ave }}\right.$ $\mathrm{d} z)_{\mathrm{ad}}=\alpha \cdot \mathrm{Di} \cdot T_{\mathrm{ave}}$ (blue curves, both left column), and the horizontally averaged RMS velocity (right column), all plotted against height $z$ at various times 


\section{Conclusions}

The numerical example presented in this study shows that the thermal state of the mantle can take geologic time to approach its statistical steady state in massive super-Earths because of the effects of adiabatic compression: When the shallow mantle is hotter than expected by the adiabatic extrapolation from the deep mantle, mantle convection temporarily occurs as a layered convection at the beginning of the calculation. It takes approximately ten Gyr in the model until this transient-layered convection yields to the whole mantle convection that occurs as the statistically steady state (see Figs. 2, 4). The transition in the convective flow pattern occurs when the cold plume's head in which the potential temperature is lower than the average potential temperature of the deep mantle descends to the layering boundary. The depth of the layering boundary is determined by hot plumes ascending from the bottom boundary. Mantle convection that currently occurs in many of massive super-Earths may be transient that may significantly affect the style of tectonic activities on these planets; whether or not plate tectonics operates, for example, is not solely determined by the size of the planet or properties of its mantle materials.

\section{Authors' contributions}

TM performed numerical simulations and the analysis of simulation data and prepared the manuscript. MK developed the numerical simulation code ACUTEMan and prepared the manuscript. MO prepared the manuscript. All authors discussed numerical simulation results. All authors read and approved the final manuscript.

\section{Author details \\ 1 Department of Deep Earth Structure and Dynamics Research, Japan Agency for Marine-Earth Science and Technology, 3173-25 Showa-machi, Kanaz- awa-ku, Yokohama, Kanagawa 236-0001, Japan. ${ }^{2}$ Geodynamics Research Center, Ehime University, 2-5 Bunkyo-cho, Matsuyama, Ehime 790-8577, Japan. ${ }^{3}$ Department of Earth Sciences and Astronomy, University of Tokyo at Komaba, 3-8-1 Komaba, Meguro, Tokyo 153-8902, Japan.}

\section{Acknowledgements}

The authors would like to thank two anonymous reviewers and the editor for their helpful comments. Numerical simulations were performed on the Earth Simulator and the supercomputer system at Japan Agency for Marine-Earth Science and Technology. This work was supported by JSPS KAKENHI Grant Numbers JP25287110, JP15H05834 and a joint research program at Geodynamics Research Center, Ehime University

\section{Competing interests}

The authors declare that they have no competing interests.

Received: 25 November 2016 Accepted: 18 March 2017 Published online: 24 March 2017

\section{References}

Borucki WJ, Koch DG, Basri G et al (2011) Characteristics of planetary candidates observed by Kepler. II. Analysis of the first four months of data. Astrophys J 736:19. doi:10.1088/0004-637X/736/1/19

Canup RM (2004) Simulations of a late lunar-forming impact. Icarus 168(2):433-456. doi:10.1016/j.icarus.2003.09.028
Chambers JE (2001) Making more terrestrial planets. Icarus 152:205-224. doi:10.1006/icar.2001.6639

Chopelas A, Boehler R (1992) Thermal expansivity in the lower mantle. Geophys Res Lett 19:1983-1986. doi:10.1029/92GL02144

Foley BJ, Bercovici D, Landuyt W (2012) The conditions for plate tectonics on super-Earths: inferences from convection models with damage. Earth Planet Sci Lett 331-332:281-290. doi:10.1016/j.epsl.2012.03.028

Jackson B, Greenberg R, Barnes R (2008) Tidal heating of extrasolar planets. Astrophys J 681:1631-1638

Kameyama M (2005) ACuTEMan: a multigrid-based mantle convection simulation code and its optimization to the Earth simulator. J Earth Simul 4:2-10

Kameyama M, Kageyama A, Sato T (2005) Multigrid iterative algorithm using pseudo-compressibility for three-dimensional mantle convection with strongly variable viscosity. J Comput Phys 206:162-181. doi:10.1016/j. jcp.2004.11.030

Kameyama M, Miyagoshi T, Ogawa M (2015) Linear analysis on the onset of thermal convection of highly compressible fluids: implications for the mantle convection of super-Earths. Geophys J Int 200:1066-1077. doi:10.1093/gji/ggu457

Kokubo E, Ida S (1998) Oligarchic growth of protoplanets. Icarus 131(1):171178. doi:10.1006/icar.1997.5840

Korenaga J (2010) On the likelihood of plate tectonics on super-earths: does size matter? Astrophys J Lett 725:L43-L46. doi:10.1088/2041-8205/725/1/ L43

Léger A, Rouan D, Schneider J et al (2009) Transiting exoplanets from the CoRoT space mission. VIII. CoRoT-7b: the first super-Earth with measured radius. Astron Astrophys 506:287-302. doi:10.1051/0004-6361/200911933

Miyagoshi T, Tachinami C, Kameyama M, Ogawa M (2014) On the vigor of mantle convection in super-Earths. Astrophys J Lett 780:L8. doi:10.1088/2041-8205/780/1/L8

Miyagoshi T, Kameyama M, Ogawa M (2015) Thermal convection and the convective regime diagram in super-Earths. J Geophys Res Planets 120:1267-1278. doi:10.1002/2015JE004793

Noack L, Breuer D (2014) Plate tectonics on rocky exoplanets: influence of initial conditions and mantle rheology. Planet Space Sci 98:41-49. doi:10.1016/j.pss.2013.06.020

Reese CC, Solomatov VS, Moresi L-N (1999) Non-Newtonian stagnant lid convection and magmatic resurfacing on Venus. Icarus 139:67-80. doi:10.1006/icar.1999.6088

Roberts JH, Barnouin OS (2012) The effect of the Caloris impact on the mantle dynamics and volcanism of Mercury. J Geophys Res 117:E02007. doi:10.1 029/2011JE003876

Roberts JH, Lillis RJ, Manga M (2009) Giant impacts on early Mars and the cessation of the Martian dynamo. J Geophys Res 114:E04009. doi:10.102 9/2008JE003287

Stamenković V, Breuer D (2014) The tectonic mode of rocky planets: part 1driving factors, models \& parameters. Icarus 234:174-193. doi:10.1016/j. icarus.2014.01.042

Stamenković V, Breuer D, Spohn T (2011) Thermal and transport properties of mantle rock at high pressure: applications to super-Earths. Icarus 216:572-596. doi:10.1016/j.icarus.2011.09.030

Stamenković V, Noack L, Breuer D, Spohn T (2012) The influence of pressuredependent viscosity on the thermal evolution of super-earths. Astrophys J 748:41. doi:10.1088/0004-637X/748/1/41

Stein C, Lowman JP, Hansen U (2013) The influence of mantle internal heating on lithospheric mobility: implications for super-Earths. Earth Planet Sci Lett 361:448-459. doi:10.1016/j.epsl.2012.11.011

Tachinami C, Senshu H, Ida S (2011) Thermal evolution and lifetime of intrinsic magnetic fields of super-earths in habitable zones. Astrophys J 726:70. doi:10.1088/0004-637X/726/2/70

Tachinami C, Ogawa M, Kameyama M (2014) Thermal convection of compressible fluid in the mantle of super-Earths. Icarus 231:377-384. doi:10.1016/j. icarus.2013.12.022

Tackley PJ, Ammann M, Brodholt JP, Dobson DP, Valencia D (2013) Mantle dynamics in super-Earths: post-perovskite rheology and self-regulation of viscosity. Icarus 225:50-61. doi:10.1016/j.icarus.2013.03.013

Tsuchiya T, Tsuchiya J (2011) Prediction of a hexagonal $\mathrm{SiO}_{2}$ phase affecting stabilities of $\mathrm{MgSiO}_{3}$ and $\mathrm{CaSiO}_{3}$ at multimegabar pressures. Proc Natl Acad Sci USA 108:1252-1255. doi:10.1073/pnas.1013594108

Turcotte DL, Schubert G (2014) Geodynamics, 3rd edn. Cambridge University Press, Cambridge 
Valencia D, O'Connell RJ (2009) Convection scaling and subduction on Earth and super-Earths. Earth Planet Sci Lett 286:492-502. doi:10.1016/j. epsl.2009.07.015

Valencia D, O'Connell RJ, Sasselov DD (2006) Internal structure of massive terrestrial planets. Icarus 181:545-554. doi:10.1016/j.icarus.2005.11.021

Valencia D, O'Connell RJ, Sasselov DD (2007) Inevitability of plate tectonics on super-Earths. Astrophys J Lett 670:L45-L48. doi:10.1086/524012 van Heck HJ, Tackley PJ (2011) Plate tectonics on super-Earths: equally or more likely than on Earth. Earth Planet Sci Lett 310:252-261. doi:10.1016/j. epsl.2011.07.029

Wetherill GW, Stewart GR (1989) Accumulation of a swarm of small planetesimals. Icarus 77(2):330-357. doi:10.1016/0019-1035(89)90093-6

\section{Submit your manuscript to a SpringerOpen ${ }^{\odot}$} journal and benefit from:

- Convenient online submission

- Rigorous peer review

- Immediate publication on acceptance

- Open access: articles freely available online

- High visibility within the field

- Retaining the copyright to your article

Submit your next manuscript at springeropen.com 